\title{
Constitutively Active MuSK Is Clustered in the Absence of Agrin and Induces Ectopic Postsynaptic-Like Membranes in Skeletal Muscle Fibers
}

\author{
Graham Jones, Chris Moore, Said Hashemolhosseini, and Hans Rudolf Brenner \\ Department of Physiology, University of Basel, CH-4051 Basel, Switzerland
}

In skeletal muscle fibers, neural agrin can direct the accumulation of acetylcholine receptors (AChR) and transcription of AChR subunit genes from the subsynaptic nuclei. Although the receptor tyrosine kinase MuSK is required for AChR clustering, it is less clear whether MuSK regulates gene transcription. To elucidate the role of MuSK in these processes, we constructed a constitutively active MuSK receptor, MuSKneuTMuSK, taking advantage of the spontaneous homodimerization of the transmembrane domain of neuT, an oncogenic variant of the neu/ erbB2 receptor. In the extrasynaptic region of innervated muscle fibers, MuSKneuTMuSK formed highly concentrated aggregates that colocalized with AChR clusters. Associated with MuSK-induced AChR clusters was a normal complement of synaptic proteins. Moreover, transcription of the AChR- $\epsilon$ subunit gene was increased, albeit via an indirect mechanism by MuSK-induced aggregation of erbB receptors and neuregu- lin. Although neural agrin was not required, the activity of MuSKneuTMuSK was nevertheless potentiated by ectopic expression of a muscle agrin isoform inactive in AChR clustering. To define the role of the kinase domain in the formation of a postsynaptic-like membrane, a second fusion receptor, neuneuTMuSK, which included the MuSK kinase but not the MuSK extracellular domain, was expressed. Significantly, neuneuTMuSK induced AChR clusters that colocalized with aggregates of endogenous MuSK. Taken together, it was concluded that the MuSK kinase domain is sufficient to initiate the recruitment of additional MuSK receptors, which then develop into highly concentrated aggregates by means of a positive feedback loop to induce a postsynaptic membrane in the absence of neural agrin.

Key words: agrin; muscle; MuSK; gene transcription; neuromuscular junction; rat
The formation of the developing neuromuscular junction (NMJ) involves the nerve-induced transcription of acetylcholine receptor (AChR) subunit genes in the subsynaptic nuclei of the muscle fiber and the aggregation of their gene products, the AChRs, at the subsynaptic membrane. Synapse formation is thought to be initiated by the release of agrin from the nerve terminal (McMahan, 1990). Agrin is expressed in several isoforms (Ruegg and Bixby, 1998). Isoforms expressed by neurons, but not those by other tissues, cluster AChRs expressed on the surface of cultured myotubes (Ruegg et al., 1992; Ferns et al., 1993) by the activation of the receptor tyrosine kinase MuSK (Glass et al., 1996). Because AChR clusters are induced independently of agrin in cultured myotubes by the addition of anti-MuSK antibodies (Xie et al., 1997; Hopf and Hoch, 1998), MuSK is likely to be part of an agrin receptor complex. However, it remains unclear whether MuSK can activate AChR gene transcription.

Synapse-specific transcription of AChR genes is thought to be induced by neuregulins (NRGs) activating erbB receptor tyrosine kinases, both of which are localized at the neuromuscular junction (for review, see Fischbach and Rosen, 1997). However, the agrin- and NRG-activated pathways may converge, because ectopic agrin can induce $\mathrm{AChR}$ gene transcription in nonsynaptic

\footnotetext{
Received Dec. 18, 1998; revised Feb. 16, 1999; accepted Feb. 19, 1999.

This work was supported by grants from the Ott-Fonds of the Swiss Academy of Medical Sciences (G.J.), the Swiss National Science Foundation, the Helmut Horten Stiftung, and the Sandoz Stiftung (H.R.B.). We thank Dr. M. A. Ruegg for agrin expression constructs and comments on this manuscript, and D. Hauser for the anti-Nsk2/MuSK antibody.

Correspondence should be addressed to Dr. H. R. Brenner, Department of Physiology, University of Basel, Vesalgasse 1, CH-4051 Basel, Switzerland. Copyright (C) 1999 Society for Neuroscience $0270-6474 / 99 / 193376-08 \$ 05.00 / 0$
}

muscle regions (Jones et al., 1997), where it also aggregates muscle-derived NRGs and erbB receptors (Meier et al., 1997, 1998a; Rimer et al., 1998). Furthermore, AChR gene transcription in cultured myotubes induced by full-length agrin is dependent on the erbB-receptor pathway (Meier et al., 1998a).

Despite the evidence equating the biological activity of neural agrin with MuSK activation in subsynaptic differentiation, several inconsistencies remain unresolved. One question is how in extrasynaptic regions of innervated muscle fibers where MuSK is reported to be absent (Valenzuela et al., 1995), subsynaptic differentiation is initiated by agrin, and which pathways induce MuSK aggregation at mature ectopic postsynaptic-like membranes (Meier et al., 1997). Second, in cultured myotubes, activation of MuSK by neural agrin added in soluble form induces AChR clustering only but not AChR gene transcription (Jones et al., 1996). Rather, the stable attachment of agrin to a laminin substrate is required for gene transcription, consistent with the idea that agrin may have roles beyond MuSK activation. For example, signaling by agrin appears to involve integrins (Martin and Sanes, 1997). Finally, the role of an agrin-induced NRG/erbB receptor pathway via activation of MuSK is unclear, because localization of erbB receptors is absent in both rapsyn- and MuSK-deficient muscle, but localized transcription appears to be absent from the latter only (Gautam et al., 1995; Moscoso et al., 1995; DeChiara et al., 1996).

These problems were addressed by expressing in muscle fibers constitutively active chimeric constructs comprising combinations of the extra- and intracellular domains of MuSK and erbB2/neu separated by the transmembrane domain of neuT, an oncogenic variant of the erbB2/neu receptor (Bargmann et al., 1986). In this 
way, the roles of MuSK in various aspects of agrin-induced differentiation of the postsynaptic muscle membrane could be examined directly.

\section{MATERIALS AND METHODS}

Construction of MuSK and erbB2/neu fusion receptors. MuSK cDNA was amplified by RT-PCR from primary rat myotube poly $\left(\mathrm{A}^{+}\right) \mathrm{RNA}$, and full-length cDNA was subcloned into the EcoRI and $X b a$ I sites of p_myc, a pcDNA1 (Invitrogen, Carlsbad, CA)-based vector that includes five copies of the myc epitope (Meier et al., 1998b) to create pMuSK_myc. Rat neuT cDNA was isolated from the vector pSVneuT (Bargmann et al., 1986). The nomenclature adopted for this paper divides the receptor cDNA into extracellular, transmembrane, and intracellular/kinase domains. To construct pMuSKneuTneu, a region of the extracellular domain of MuSK between nucleotides 1416 and 1596 was amplified using the following PCR primers: MuSK1416_s, 5' -ggagtgcagcaagcttcccagc-3', which includes the HindIII site at position 1426, and MuSK1596 asNdeI 5'ccgggcacatatgcaggcgagacggcgaaggaagacgtgga-3' (Microsynth, Balgach, Switzerland), which introduces an NdeI site (underlined) encoding a linker peptide AYV between the MuSK extracellular domain and the neuT transmembrane domain in the completed fusion receptor. The transmembrane domain and part of the intracellular domain of neuT was amplified using the following oligonucleotides: neuT1985_sNdeI 5'ccggggcatatgtgacattcatcattgcaactgtagagggcgtc- $3^{\prime}$, which introduces an NdeI site and neuT2406_as 5'-gatgtcaggcagatgccc-3'. The resulting PCR product was digested with $N d e \mathrm{I}$ and $\mathrm{XbaI}$ (at nucleotide 2332) and subcloned with the HindIII- and NdeI-digested MuSK PCR product into pMuSK myc digested with HindIII and $X b a \mathrm{I}$. This fragment was then excised by digestion with EcoRI and $X b a \mathrm{I}$ and ligated with the intracellular region of neuT by digesting pSVneuT with $X b a \mathrm{I}$ and $X h o \mathrm{I}$ and subcloning into pCDNAI digested with EcoRI and XhoI. To construct pMuSKneuTMuSK_myc, the following PCR amplifications were performed: pMuSKneuTneu was amplified using MuSK1416_s and MuSKneuT_as 5'-cctccttcggcagcaatagattaggattccaacgacc-3', which included MuSK intracellular domain (underlined) and neuT transmembrane sequences; intracellular domains of MuSK were amplified using MuSK1663 s 5'-tattgctgccgaaggagg-3', which includes the same region as underlined in MuSKneuT_as and MuSK2727_as. The two PCR products were diluted, mixed, and reamplified using the flanking primers MuSK1416_s and MuSK2727_as. The resulting product was isolated and digested with HindIII and $\overline{B l} p \mathrm{I}$ (at position 1812) and subcloned into pMuSK_myc digested with HindIII and BlpI. To construct pneuneuTMuSK, a fragment of neuT extending from the extracellular domain into the neuT transmembrane domain was amplified using the primers neu1886_s, 5'-gaggagggcatatgccag-3' and neuTM.Glu_as, 5' -ggacgccctctacagttgc- $-3^{\prime}$; the neuT transmembrane domain into the MuSK intracellular domain was amplified using the primers neuTM.Glu_s, 5'-gcaactgtagagggcgtcc- $3^{\prime}$, and MuSK1921 as, 5'-cctccttcagcatcttcacagccacc-3'. The resulting PCR products were diluted, mixed, and reamplified using neu1886_s and MuSK as. The resulting PCR product was digested with the restriction endonucleases $N d e \mathrm{I}$ and $B l p \mathrm{I}$ and combined with pMuSK_myc digested with HindIII and BlpI and an neuT extracellular fragment HindIII/NdeI. The resulting constructs were then sequenced, and equivalent expression of MuSKneuTMuSK, MuSKneuTneu, and neuneuTMuSK was confirmed by immunoprecipitation and Western blot analysis of transfected Cos-1 cells.

Construction of pGFP-S6. To identify transfected myoblasts and injected muscle fibers, we constructed a green fluorescent protein (GFP) (Clontech, Palo Alto, CA) that was targeted into the nucleus. To do this, three nuclear localization signals (NLSs) present at the C terminus of the S6 protein (Schmidt et al., 1995) were fused to the C terminus of humanized GFP. An EcoRI restriction endonuclease site was introduced via PCR at the $3^{\prime}$ end of cDNA encoding humanized GFP, which was then cloned 5' HindIII-EcoRI 3' into the vector pCMX-pI2 (Umesono et al., 1991). A $251 \mathrm{bp}$ fragment of the cDNA encoding the three NLSs of protein S6 was amplified using the following primers: S6F 5'- aaagaattcaagaaacctaggaccaaagca-3', S6R 5'-tcaggatccctatttctgactggattcaga-3'. S6F includes the first NLS of S6, and S6R includes a stop codon. The resulting PCR product was digested with EcoRI and Bam HI (underlined in S6F and S6R) and subcloned into the Eco RI-BamHI sites of pCMX-pI2/GFP to generate pGFP-S6.

Transfection of $C 2 C 12$ myoblasts. For analysis of transcriptional activity of the $-216 \epsilon$ subunit gene promoter (Jones et al., 1996), $1 \times 10^{5} \mathrm{C} 2 \mathrm{C} 12$ cells were passaged onto six-well plates that had been coated with either laminin alone or laminin with agrin as described (Meier et al., 1998a). Myoblasts were transfected with $800 \mathrm{ng}$ of reporter plasmid DNA, $200 \mathrm{ng}$ of the appropriate receptor plasmid, and $200 \mathrm{ng}$ of pCH110 (Pharmacia BioTech Europe) using $5 \mu \mathrm{l}$ FuGENE 6 (Roche Diagnostics Boehringer Mannheim, Mannheim, Germany) according to the manufacturer's instructions. Myotubes were analyzed $5 \mathrm{~d}$ after transfection. Results are averages of three dishes each from at least three independent transfections. For immunoprecipitation experiments, $\mathrm{C} 2 \mathrm{C} 12$ myoblasts were transfected with $1 \mu \mathrm{g}$ of the appropriate plasmid expression vector and allowed to differentiate for $5 \mathrm{~d}$. Cells were lysed, and membrane proteins were immunoprecipitated as described (Meier et al., 1998a).

Injection of muscle fibers and immunohistochemistry. Single rat soleus fibers were injected as described previously (Jones et al., 1997; Meier et al., 1997) with $80 \mathrm{ng} / \mu \mathrm{l}$ pGFP-S6, $100 \mathrm{ng} / \mu \mathrm{l}$ pMuSKneuTMuSK_myc or pneuneuTMuSK_myc, and where applicable, $100 \mathrm{ng} / \mu \mathrm{l} \mathrm{pcAgrin}_{7 \mathrm{A0B} 0}$. Rats were killed $\overline{4}$ weeks later. For immunohistochemistry, muscles were frozen in prechilled isopentane and cut into $12 \mu \mathrm{m}$ cross sections. Immunohistochemistry of unfixed cross sections was performed using antibodies and methods as described (Meier et al., 1997).

Quantitative TaqMan fluorescent real time PCR analysis of AChR- $\epsilon$ subunit RNA in injected muscles. Expression of GFP-S6 allowed direct visualization of injected fibers, the borders of which were marked before freezing. The injected and noninjected regions were then excised, and total RNA was extracted using the Qiagen RNeasy RNA extraction kit (Qiagen AG, Basel, Switzerland). First-strand cDNA was synthesized from $2 \mu \mathrm{g}$ of total RNA primed with $250 \mathrm{ng}$ of random hexamers (Roche Diagnostics Boehringer Mannheim) using Superscript reverse transcriptase (Life Technologies, Gaithersburg, MD). First-strand cDNA was purified using the High Pure PCR Product Purification Kit (Roche Diagnostics Boehringer Mannheim). The primers $\beta$-actin sense $5^{\prime}$ ttcaacaccccagccatgt- $3^{\prime}$, anti-sense $5^{\prime}$-gtggtacgaccagaggcataca- $3^{\prime}$, AChR- $\epsilon$ subunit sense $5^{\prime}$-ccaacgactcacgccacat-3', and anti-sense 5 '-ggcgcggcagtagctcta- $3^{\prime}$ were synthesized and flanked the TaqMan oligonucleotide probes $\beta$-actin, $5^{\prime}$-(FAM)cgtagccatccaggctgtgttgtcc(TAMRA)-3' and AChR- $\epsilon, 5^{\prime}-($ FAM) ccctcggetgcgccagatttt(TAMRA)-3'. TaqMan PCR was performed using the TaqMan PCR Core Reagent Kit as described by the manufacturer (Perkin-Elmer Applied Biosystems), with the exception that $\mathrm{MgCl}_{2}$ was increased to $5 \mathrm{~mm}$. AChR- $\epsilon$ subunit mRNA was quantitated relative to $\beta$-actin mRNA using the comparative $C_{\mathrm{T}}$ method. $C_{\mathrm{T}}$ (threshold cycle) is defined as the cycle number at which the amount of amplified target passes a fixed threshold above baseline. $\Delta C_{\mathrm{T}}$ is the difference in threshold cycles between AChR- $\epsilon$ and $\beta$-actin, calculated for RNA isolated from either injected or noninjected regions. Subtracting the $\Delta C_{\mathrm{T}}$ of the noninjected region from the $\Delta C_{\mathrm{T}}$ of the injected region gives the value $\Delta \Delta C_{\mathrm{T}}$, which when applied in the formula $2^{-\Delta \Delta}$ CT , gives the amount of AChR- $\epsilon$ subunit RNA in injected muscle fibers, normalized to $\beta$-actin, and relative to the noninjected region. To ensure that the $\Delta \Delta C_{\mathrm{T}}$ calculation was valid, the efficiency with which both genes were amplified was examined and found to be the same.

\section{RESULTS}

\section{Constitutive activation of MuSK induces ectopic AChR clusters}

To examine the biological activity of MuSK independently of activation by agrin, we designed an activated MuSK receptor construct. This was performed by exploiting the neuT oncogene, in which a single point mutation in the transmembrane domain results in the spontaneous formation of active neuT homodimers. It is now apparent that although the transmembrane domain of the receptor tyrosine kinases is required for activity, correct biological signaling also involves dimerization of the extracellular and kinase domains (Siegel and Muller, 1996; Burke et al., 1997; Tzahar et al., 1997). On the basis of these findings, we reasoned that a MuSK receptor fusion construct, MuSKneuTMuSK, in which the MuSK extracellular and kinase domains were separated by a neuT transmembrane domain (for the nomenclature of the fusion constructs, see Fig. 1), would homodimerize yet retain MuSK biological activity.

In response to neural agrin, the MuSK kinase domain is only transiently phosphorylated over a time course of minutes (Glass et al., 1996), and a similar problem occurs with constitutive 
extracellular TM intracellular

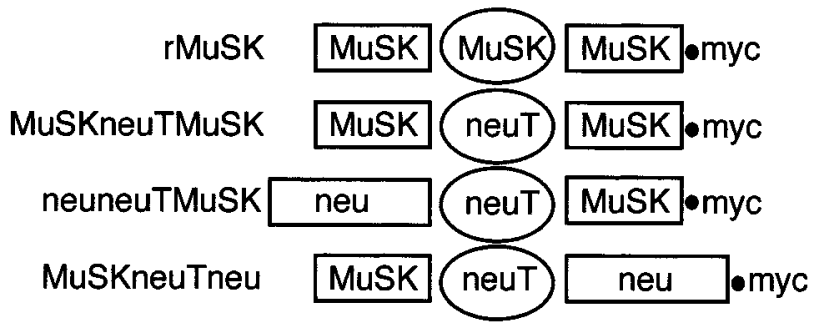

Figure 1. Schematic of MuSK and neu/erbB2 receptor fusion constructs. The nomenclature adopted divides each receptor tyrosine kinase into extracellular, transmembrane $(T M)$, and intracellular/kinase domains. Also shown is the C-terminal myc tag. The plasmid encoding each chimeric receptor is denoted by the prefix "p" and myc as suffix. Wild-type MuSK $(r M u S K)$ is indicated as are each of the fusion receptor products encoded by each plasmid: MuSKnеuTMuSK, MuSKnеuTneu, and пеипеиTMuSK.

activation of the MuSK kinase domain. Therefore, because only those isoforms of agrin that induce AChR clustering also phosphorylate MuSK (Glass et al., 1996), AChR clustering in C2C12 myotubes was used to assay MuSKneuTMuSK activity. C2C12 myoblasts were transfected with pMuSKneuTMuSK_myc and pGFP-S6, which encodes nuclear-localized GFP. ACh $\bar{R}$ clusters were induced on $80 \%$ of GFP-positive myotubes, which compared favorably with AChR clustering on myotubes transfected with pGFP-S6 but treated with neural agrin (94\% AChR cluster/GFP colocalization). In contrast, in myoblasts transfected with pMuSKneuTneu_myc, which consists of the MuSK extracellular domain but the erbB2/neu kinase domain, only 3\% of GFPpositive myotubes exhibited AChR clustering, the same as that seen for untreated myotubes transfected with pGFP-S6 alone (data not shown).

We next investigated whether activation of MuSK was sufficient to induce AChR clusters in the nerve-free region of innervated muscle fibers. To identify injected fibers, pMuSKneuTMuSK_myc was coinjected with pGFP-S6. Overexpression of constitutively active MuSKneuTMuSK induced approximately one AChR cluster per injected fiber [Fig. 2, MuSKneuTMuSK, and right panel, - cAgrin 7AOBO (1.2 \pm 0.1 AChR clusters/injected fiber; SEM, $n=9$ muscles/200 fibers)]; they were similar in number and appearance to those induced by low levels of neural agrin. Similar findings were obtained with a chimera lacking a myc tag (data not shown). In contrast, ectopic expression of wild-type MuSK only rarely induced AChR clusters, which were small and point-like in appearance (data not shown).

It was shown previously that the distribution of endogenous MuSK is coextensive with that of ectopic agrin deposits, independent of whether an active or an inactive isoform of agrin was expressed (Meier et al., 1997; D. M. Hauser and M. A. Ruegg, personal communication). This suggests that agrin may influence AChR clustering via two mechanisms: (1) by accumulating MuSK and (2) by phosphorylating MuSK. We were therefore interested to see whether an inactive muscle isoform of chick agrin influenced the appearance of MuSKneuTMuSK and/or AChR clusters. To do this, expression plasmids encoding either MuSKneuTMuSK or chick agrin, cAgrin $_{7 \mathrm{AOB} 0}$, were injected into neighboring muscle fibers. Although the morphology of the resulting AChR clusters remained similar to those induced by MuSKneuTMuSK alone (Fig. 2, MuSKneuTMuSK/cAgrin), cAgrin $_{7 \mathrm{A0в0}}$ increased the average number of AChR clusters on pMuSKneuTMuSK_myc-

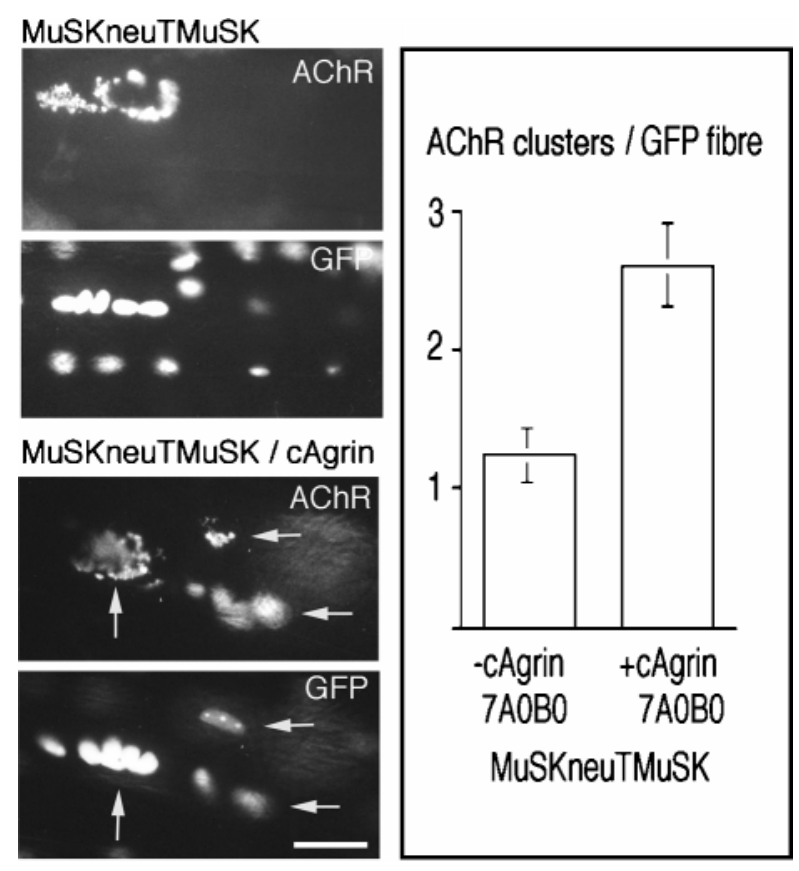

Figure 2. Constitutive activation of MuSK is sufficient to induce ectopic, activity-resistant AChR clusters but is enhanced by a muscle agrin isoform, cAgrin ${ }_{7 \mathrm{~A} 0 \mathrm{~B} 0}$. In muscle fibers injected with pMuSKneuTMuSK_myc, generally one AChR cluster was recorded per injected fiber (MuSKneuTMuSK, $A C h R$ ) and was associated with clusters of GFP-positive nuclei $(G F P)$. cAgrin ${ }_{7 \mathrm{~A} 0 \mathrm{~B} 0}$ secreted from neighboring fibers increased the number of AChR clusters on pMuSKneuTMuSK myc-injected fibers (MuSKneuTMuSK/cAgrin). In the example shown, three AChR clusters (arrows) are present in close proximity on the same injected fiber as seen by colocalization with clusters of GFP-positive nuclei. Analysis of the average number of $\mathrm{AChR}$ clusters per GFP-positive fiber showed that cAgrin $_{7 \mathrm{A0B} 0}$ increased the average number of MuSKneuTMuSK-induced AChR clusters per injected fiber more than twofold (right panel, + cAgrin 7AOB0; SEM, $n=4$ muscles, 100 fibers) over that observed in muscle fibers injected with pMuSKneuTMuSK_myc alone (right panel, -cAgrin 7AOB0; SEM, $n=9$ muscles, 200 fibers). Scale bar, $50 \mu \mathrm{m}$.

injected fibers more than twofold, such that several AChR clusters were now present on each injected fiber examined [Fig. 2, right panel, +cAgrin $7 A 0 B 0(2.6 \pm 0.3 \mathrm{AChR}$ clusters/injected fiber; SEM, $n=4$ muscles/100 fibers)]. Thus, MuSKneuTMuSK induced ectopic AChR clusters independently of neural agrin, but this activity was enhanced by exogenous chick muscle agrin cAgrin $_{\text {7А0B0 }}$

\section{Activation of the MuSK kinase domain initiates recruitment and aggregation of additional MuSK receptors}

Endogenous MuSK colocalizes with ectopic neural (Meier et al., 1997) and muscle agrin isoforms (Hauser and Ruegg, personal communication), suggesting that agrin may be involved in the localization of MuSK to the NMJ. To examine the distribution of MuSKneuTMuSK in the absence of agrin, fibers injected with pMuSKneuTMuSK_myc were analyzed in frozen cross sections through the injected region. First, the distribution of MuSKneuTMuSK was determined by immunohistochemistry using antibodies that recognized the extracellular domain of MuSK (Meier et al., 1997) or the C-terminal myc tag. Intense myc immunoreactivity colocalized with AChR clusters, with only faint staining extending throughout the cytoplasm and around the remainder of the muscle fiber circumference (Fig. 3A, MuSKneuTMuSK, myc). MuSK immunoreactivity was similarly concentrated at AChR 


\section{A. MuSKneuTMuSK}

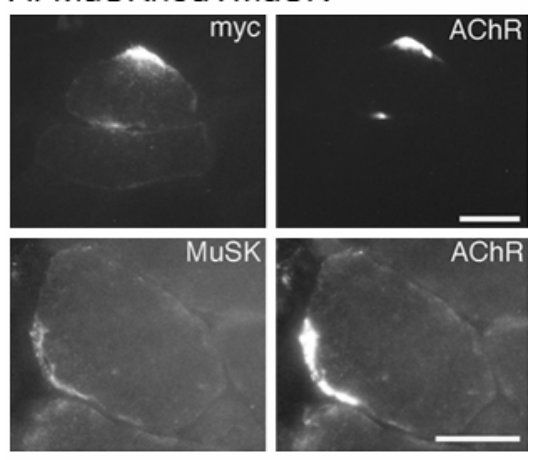

B. neuneuTMuSK
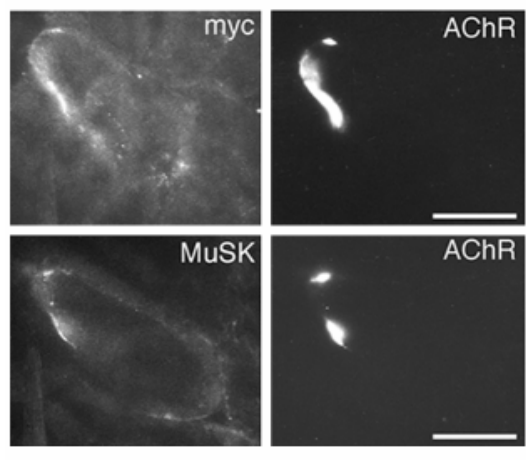

\begin{tabular}{|ll|}
\hline Antibody & \multicolumn{1}{c|}{ Target } \\
$\alpha-$ myc & MuSKneuTMuSK \\
& neuneuTMuSK \\
$\alpha-$ MuSK & endogenous MuSK \\
& MuSKneuTMuSK \\
\hline
\end{tabular}

Figure 3. A, Distribution of AChRs and MuSKneuTMuSK in injected fibers. MuSKneuTMuSK was identified using an antibody that recognized a C-terminal myc epitope (myc) (bottom panel). Highly concentrated deposits of MuSKneuTMuSK colocalized with AChR clusters $(A C h R)$. Similarly, by using an antibody that recognized the extracellular domain of MuSK, MuSK immunoreactivity was restricted to AChR clusters $(M u S K) . B$, Ectopic expression of neuneuTMuSK, which includes the MuSK kinase domain but not the MuSK extracellular domain, recruits endogenous MuSK and induces AChR clusters in innervated muscle fibers. AChR clusters induced by neuneuTMuSK $(A C h R)$ colocalize with neuneuTMuSK $(m y c)$ and endogenous MuSK $(M u S K)$, resolved using either anti-myc or anti-MuSK (extracellular domain) antibodies, respectively. Bottom panel, Summary of receptor recognition by anti-myc and anti-MuSK antibodies. Scale bar, $50 \mu \mathrm{m}$.

clusters (Fig. 3A, MuSKneuTMuSK, MuSK). In muscles injected with pMuSKneuTMuSK_myc and pcAgrin ${ }_{7 \text { Аов0 }}$, concentrated aggregates of MuSKneuTMuSK still colocalized with AChRs (data not shown). The formation of highly concentrated aggregates of MuSKneuTMuSK coincident with AChR clusters, both in the presence and absence of cAgrin $_{7 \mathrm{A0B} 0}$, thus implies a positive feedback loop, driven by the activation of MuSK itself. Furthermore, because MuSKneuTneu did not induce AChR clustering in injected muscle fibers (data not shown), the MuSK kinase domain was essential for this process. To directly test whether activation of the MuSK kinase was sufficient for the recruitment of MuSK, we constructed pneuneuTMuSK_myc, which includes the erbB2/neu extracellular domain and the MuSK

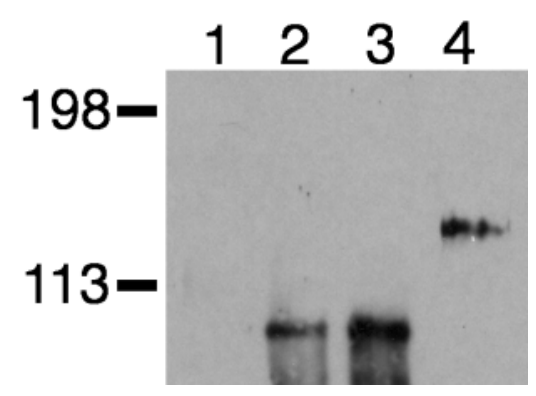

Figure 4. neuneuTMuSK interacts with endogenous MuSK receptors in $\mathrm{C} 2 \mathrm{C} 12$ myotubes. Lysates from $\mathrm{C} 2 \mathrm{C} 12$ myotubes transfected with pGFP-S6 alone (lane 1), pneuneuTMuSK myc (lane 2), pMuSKneuTMuSK_myc (lane 3), or pMuSKneuTneu_myc (lane 4) were immunoprecipitated with anti-myc antibody. Immunoprecipitated proteins were analyzed by Western blotting by incubation with an anti-MuSK antibody that recognized the extracellular domain of MuSK. Molecular weight markers indicated in kilodaltons.

kinase domain (Fig. 1). In contrast to MuSKneuTMuSK, neuneuTMuSK did not induce AChR clusters in C2C12 myotubes (data not shown), similar to a trkC/MuSK chimera, which also lacks the MuSK extracellular domain and is consistent with the idea that the MuSK extracellular domain is required for AChR clustering (Glass et al., 1997). Surprisingly, however, ectopic expression of neuneuTMuSK in innervated muscle fibers induced AChR clusters indistinguishable from those induced by MuSKneuTMuSK (Fig. 3B, neuneuTMuSK, $A C h R$ ), suggesting that the MuSK extracellular domain must be supplied by endogenous MuSK. Indeed, both neuneuTMuSK, identified specifically by an anti-myc antibody (Fig. 3B, neuneuTMuSK, myc), and endogenous MuSK, identified by an antibody against the extracellular domain of MuSK (Fig. 3B, neuneuTMuSK, MuSK) (Meier et al., 1997), colocalized with AChR clusters.

The association of neuneuTMuSK with endogenous MuSK receptors was confirmed by immunoprecipitation experiments. Extracts from $\mathrm{C} 2 \mathrm{C} 12$ myotubes transfected with pneuneuTMuSK_myc were immunoprecipitated with an anti-myc antibody and analyzed by Western blotting using an anti-MuSK antibody that does not recognize neuneuTMuSK (Fig. 4, lane 2). A single band of $100 \mathrm{kDa}$ was detected, corresponding to endogenous MuSK. A similar result is shown for C2C12 myotubes transfected with pMuSKneuTMuSK_myc (Fig. 4, lane 3). However, in myotubes transfected with pMuSKneuTneu_myc, no endogenous MuSK was co-immunoprecipitated. Instead, only MuSKneuTneu $(120 \mathrm{kDa})$ was detected using an anti-MuSK antibody (Fig. 4, lane 4 ). Furthermore, because neither endogenous erbB2 nor erbB3 receptors were co-immunoprecipitated with neuneuTMuSK or MuSKneuTneu (data not shown), the chimeric receptors primarily formed homodimers. Together, these results suggested that activation of the MuSK kinase domain was required for the aggregation of endogenous MuSK receptors seen at ectopic AChR clusters.

\section{MuSKneuTMuSK induces a postsynaptic membrane that includes a normal complement of synaptic proteins}

Rapsyn, phosphotyrosine, and utrophin each colocalized with AChR clusters induced by MuSKneuTMuSK (data not shown). MuSKneuTMuSK also recruited laminin- $\beta 2$ (Fig. 5, lam- $\beta 2$ ), a synaptic component of the extracellular matrix potentially important in the differentiation of the presynaptic nerve terminal (Porter et al., 1995; Patton et al., 1997). Also present at ectopic AChR 

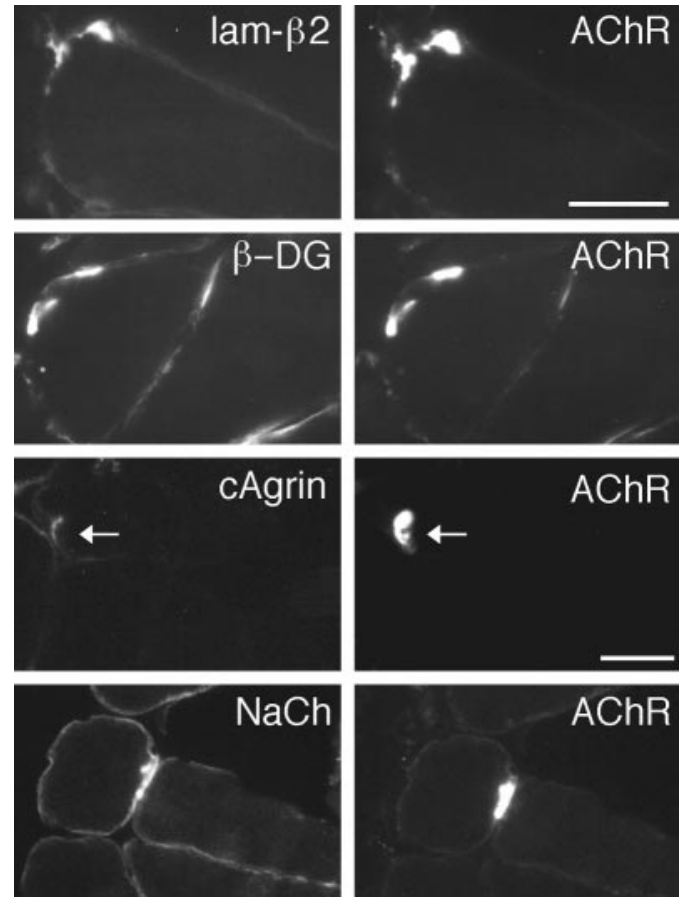

Figure 5. MuSKneuTMuSK-induced AChR clusters include a normal complement of postsynaptic proteins. AChR clusters were identified $(A C h R)$ and examined for the presence of laminin- $\beta 2$ (lam- $\beta 2)$, $\beta$-dystroglycan $(\beta-D G)$, and $N a C h$. In muscles injected with pMuSKneuTMuSK and pcAgrin ${ }_{7 \mathrm{~A} 0 \mathrm{~B} 0}$, deposits of $\mathrm{cAgrin}_{7 \mathrm{A0 \textrm {B }} 0}$ at AChR clusters were observed (cAgrin, arrow). Scale bar, $50 \mu \mathrm{m}$.

clusters was the postsynaptic membrane protein $\beta$-dystroglycan (Fig. 5, $\beta$-DG). $\beta$-Dystroglycan binds noncovalently with the extracellular glycoprotein $\alpha$-dystroglycan, and both are integral components of the dystrophin-associated protein complex (Tinsley et al., 1994). In addition, both neural and muscle agrin bind $\alpha$-dystroglycan via domains not required for MuSK activation (Gesemann et al., 1996). In muscle fibers expressing MuSKneuTMuSK, no accumulation of endogenous muscle agrin was seen at MuSKneuTMuSK-induced AChR clusters (data not shown; see also Meier et al., 1997). However, ectopic expression of chick muscle agrin cAgrin $_{7 \mathrm{A0B} 0}$ resulted in deposits of $\mathrm{cAgrin}_{7 \mathrm{A0B0}}$ at AChR clusters in neighboring pMuSKneuTMuSK_myc-injected fibers (Fig. 5, cAgrin). Thus, additional binding sites for agrin are present at MuSKneuTMuSK-induced AChR clusters.

We also examined whether voltage-activated $\mathrm{Na}^{+}$-channels (NaChs) were present. Unlike the AChR channels, which aggregate early in synapse formation and become localized to the crests of the postsynaptic folds (Fertuck and Salpeter, 1976), NaChs aggregate late in the maturation of the postsynaptic membrane (Lupa et al., 1993), and they are concentrated in the troughs of the postsynaptic folds (Boudier et al., 1992), suggesting that the aggregation and localization of $\mathrm{NaChs}$ are regulated differently to the AChRs (Colledge and Froehner, 1998). However, NaChs also colocalized with MuSKneuTMuSK-induced AChR clusters (Fig. 5, $\mathrm{NaCh}$ ). Therefore, clustering of $\mathrm{NaChs}$ is also regulated by MuSK.

\section{Activation of MuSK aggregates erbB receptors and NRGs}

We next asked whether MuSK, in the absence of neural agrin, induced the assembly of the erbB/NRG signaling pathway. In-
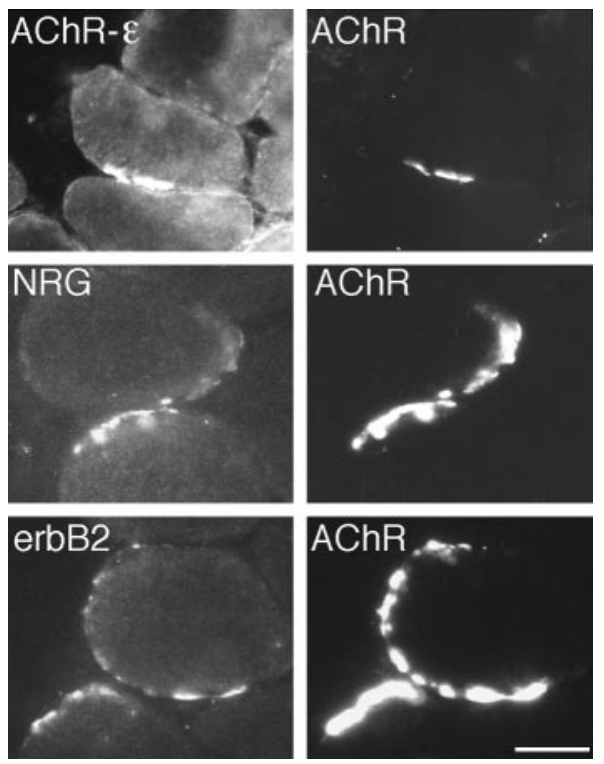

Figure 6. MuSKneuTMuSK-induced AChR clusters include the AChR- $\epsilon$ subunit and colocalize with the erbB2 receptor tyrosine kinase and NRGs. AChR clusters were identified $(A C h R)$ and examined for the presence of the AChR- $\epsilon$ subunit $(A C h R-\epsilon)$, NRGs $(N R G)$, or erbB2 (erbB2). Scale bar, $50 \mu \mathrm{m}$.

deed, using an antibody specific for the mammalian AChR- $\epsilon$ subunit, we found that AChR clusters were of the adult AChR subtype (Fig. 6, AChR- $\epsilon$ ) and that such AChR clusters contained aggregates of NRGs (Fig. 6, NRG), erbB2 (Fig. 6, erbB2), and erbB3 receptors (data not shown). To ascertain whether this was reflected in increased gene transcription, AChR- $\epsilon$ subunit mRNA from the region of injected muscle expressing MuSKneuTMuSK was quantified using a real-time RT-PCR assay. To increase the sensitivity of this assay, we used muscles that had been co-injected with pMuSKneuTMuSK_myc and pcAgrin ${ }_{7 \text { Аово }}$. Ectopic expression of pcAgrin ${ }_{7 \mathrm{A0B} 0}$ alone did not increase AChR- $\epsilon$ subunit mRNA in the injected region $(1.15 \pm 0.1$, SEM; $n=3$ muscles), whereas expression of pMuSKneuTMuSK_myc and pcAgrin $_{7 \text { Аово }}$ increased AChR- $\epsilon$ subunit mRNA by up to fourfold (3.8 $\pm 0.3, \mathrm{SEM} ; n=3$ muscles). The close agreement between these results and those from similar experiments using neural agrin (Jones et al., 1997; Meier et al., 1997; Rimer et al., 1998) is consistent with the hypothesis that MuSKneuTMuSK activates AChR gene transcription by organizing the erbB/NRG pathway.

We next sought to test this hypothesis further in cultured $\mathrm{C} 2 \mathrm{C} 12$ myotubes, which have proved to be a convenient and reliable model for examining the regulation of AChR gene transcription by agrin (Jones et al., 1996; Meier et al., 1998a). Initially, we compared the transcriptional activation imparted by activation of either the MuSK or erbB2/neu kinase domains. For this purpose, an AChR- $\epsilon$ subunit promoter reporter plasmid, pLCF216 $\epsilon$ (Jones et al., 1996), was cotransfected with either pMuSKneuTneu_myc or pMuSKneuTMuSK_myc. Surprisingly, unlike in innervated muscle fibers, no significant increase in luciferase activity was induced by MuSKneuTMuSK (Fig. 7, MuSK). In contrast, MuSKneuTneu clearly activated transcription, with luciferase activity increased approximately fivefold over that seen for MuSKneuTMuSK or in control cultures (Fig. 7, neu).

The role of MuSK in neural agrin-induced AChR transcription in cultured $\mathrm{C} 2 \mathrm{C} 12$ myotubes was therefore reexamined using a 


\section{Relative luciferase activity $-216 \varepsilon$}

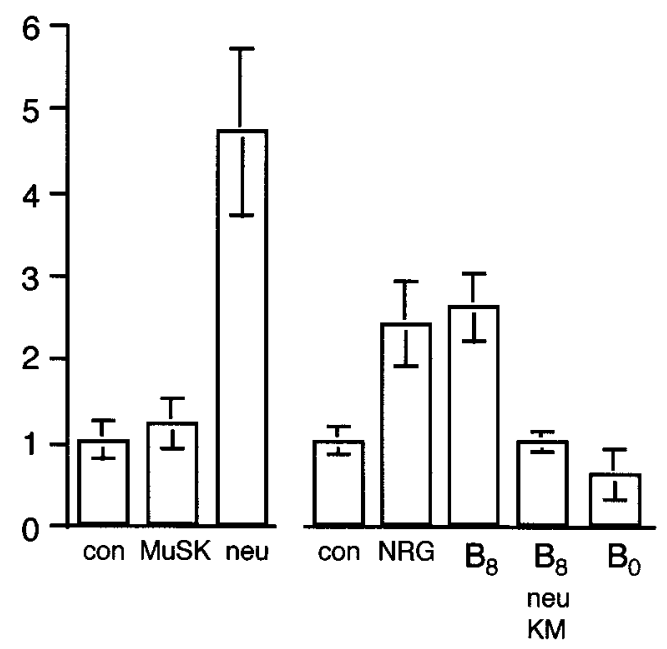

Figure 7. MuSK does not directly increase transcription of the AChR- $\epsilon$ subunit gene in $\mathrm{C} 2 \mathrm{C} 12$ myotubes. The transcriptional activity of the MuSK and erbB2/neu kinase domains was directly compared by culturing $\mathrm{C} 2 \mathrm{C} 12$ myoblasts on a laminin substrate. Myoblasts were transfected with either pLCF216 $\epsilon$ (control: con) or pLCF216 $\epsilon$ with pMuSKneuTMuSK_myc $(\mathrm{MuSK})$ or pMuSKneuTneu_myc (neu), a chimeric receptor that included the erbB2/neu kinase domain. The role of MuSK in AChR transcription was investigated further by culturing $\mathrm{C} 2 \mathrm{C} 12$ myoblasts on a substrate of neural mini-agrin $\mathrm{cN} 25_{7} \mathrm{C} 21_{\mathrm{B} 8}$ adhered to laminin $\left(B_{8}\right)$. The requirement for erbB2/neu was tested by cotransfecting a dominant-negative erbB2/ neu mutant into myoblasts similarly cultured on a laminin $/ \mathrm{cN} 25_{7} \mathrm{C} 21_{\mathrm{B} 8}$ substrate $\left(B_{8}\right.$, neu $\left.K M\right)$. Mini-agrin $\mathrm{cN} 25_{7} \mathrm{C} 21_{\mathrm{B} 0}\left(B_{0}\right)$, which does not phosphorylate MuSK or induce AChR clustering, did not increase transcription. Also shown is the transcriptional activity of a saturating amount of soluble NRG $(N R G)$.

truncated form of neural agrin, mini-agrin $\mathrm{cN} 25_{7} \mathrm{C} 21_{\mathrm{B} 8}$. In $\mathrm{cN} 25_{7} \mathrm{C} 21_{\mathrm{B} 8}$, the $\mathrm{N}$-terminal laminin-binding domain of agrin is linked to a minimal C-terminal $21 \mathrm{kDa}$ fragment that is sufficient for MuSK phosphorylation, AChR clustering, and binding of the putative agrin receptor (Gesemann et al., 1995, 1996; Meier et al., 1998b). In innervated muscle fibers, $\mathrm{cN} 25_{7} \mathrm{C} 21_{\mathrm{B} 8}$ induces AChR- $\epsilon$ subunit gene transcription as well as aggregation of NRG and erbB2 (Meier et al., 1998b). Importantly, in contrast to full-length neural agrin, the truncated mini-agrin lacks the $\mathrm{N}$-terminal heparan sulfate glycosaminoglycan (GAG) side chains that are required for binding of NRG (Meier et al., 1998a), thus excluding transcriptional activation independent of MuSK activation via GAG-attached NRGs.

As with full-length agrin (Jones et al., 1997), mini-agrin $\mathrm{cN} 25_{7} \mathrm{C} 21_{\mathrm{B} 8}$ bound to a laminin substrate (Fig. 7, $B_{8}$ ), but not when added directly to the culture supernatant (data not shown), increased AChR- $\epsilon$ subunit promoter activity in a quantitatively similar manner to MuSKneuTneu (Fig. 7, neu) or saturating NRG (Fig. 7, NRG). The induction of transcription by mini-agrin $\mathrm{cN} 25_{7} \mathrm{C} 21_{\mathrm{B} 8}$ was abolished by the expression of a dominant negative erbB2/neu receptor (Fig. 7, $B_{8}$ neu $K M$ ), which inhibits NRG-dependent activation of intracellular pathways (Wallasch et al., 1995). In contrast, an inactive mini-agrin isoform, $\mathrm{cN} 25_{7} \mathrm{C} 21 \mathrm{~B}_{0}$, which does not induce $\mathrm{AChR}$ clustering, failed to increase $\mathrm{AChR}-\epsilon$ subunit gene transcription (Fig. 7, $B_{0}$ ). Thus, in C2C12 myotubes, activated MuSK was not sufficient by itself to activate AChR gene transcription directly. Instead, transcription activated by MuSK required the NRG/erbB pathway, dependent on interaction with substrate-bound agrin or a related molecule, a condition that in innervated muscle fibers may be met by a native basal lamina component.

\section{DISCUSSION \\ MuSK kinase domain activation recruits additional MuSK receptors}

It is thought that a prerequisite for the assembly of the postsynaptic muscle membrane is the formation of a MuSK scaffold (Apel et al., 1997; Glass and Yancopoulos, 1997). The observation that MuSKneuTMuSK was not distributed evenly around the circumference of the muscle fiber, but instead was concentrated at AChR clusters independently of agrin, showed that the formation of such a MuSK scaffold is an activity intrinsic to active MuSK. Previous reports have shown that the MuSK ectodomain interacts with rapsyn (Apel et al., 1997), whereas the kinase domain is required for phosphorylation of the AChR- $\beta$ subunit (Glass et al., 1997), and that both domains are required for AChR clustering. In results presented here, a chimeric receptor lacking the MuSK ectodomain, neuneuTMuSK, induced ectopic AChR clusters by recruiting endogenous MuSK receptors. Therefore, the primary event in the recruitment of MuSK to a developing scaffold is the activation of the kinase domain, which, given that these experiments were done in the extra-synaptic region of innervated muscle fibers, precedes the formation of AChR clusters. The recruitment of MuSK via a positive feedback loop might be particularly important for the induction by neural agrin of ectopic AChR clusters in the extra-synaptic region of innervated muscles fibers, where MuSK expression is low but not absent (C. Moore and H. R. Brenner, unpublished observations).

The reasons for the failure of neuneuTMuSK to induce AChR clusters in $\mathrm{C} 2 \mathrm{C} 12$ myotubes remain unclear. One possible reason is that the extracellular matrix differs between cultured $\mathrm{C} 2 \mathrm{C} 12$ myotubes and innervated muscle fibers. In addition, it should also be remembered that direct injection of plasmid DNA is an extremely efficient method of transfecting muscle fibers. This might be important in achieving a concentrated point source of neuneuTMuSK, thereby facilitating the recruitment of endogenous MuSK receptors and the formation of AChR clusters.

The ligand-independent activation of MuSK described here is similar to the formation of active MuSK homodimers by activating antibodies (Xie et al., 1997; Hopf and Hoch, 1998). It could also explain the occasional small AChR clusters on a low number of myofibers in mice deficient for agrin (Gautam et al., 1996), arising through spontaneous association of MuSK homodimers that in the absence of agrin would occur only with low frequency. The formation of such foci of endogenous MuSK could be promoted by the elevated level of MuSK expression in fetal and neonatal myotubes (Valenzuela et al., 1995; Bowen et al., 1998). It is possible that in denervated muscle, the formation of dense patches of AChR clusters (Ko et al., 1977) is attributable to increased levels of MuSK, which leads to the ligand-independent formation of active homodimers.

Activation of MuSK in injected muscle fibers was sufficient to induce localized deposits of laminin- $\beta 2$. Interestingly, no accompanying accumulation of endogenous muscle agrin was observed. Thus, because laminin present in the muscle basal lamina binds agrin (Denzer et al., 1997), this indicates that laminin- $\beta 2$ is aggregated at the NMJ independently of its binding to agrin. In the context of laminin $11(\alpha 5 \beta 2 \gamma 1)$, laminin- $\beta 2$ is a stop signal for motor neurons at the NMJ (Patton et al., 1997). This would explain why, in agrin-deficient mice, innervating neurons do sometimes stop and differentiate at sites opposing agrin- 
independent AChR clusters (Gautam et al., 1996), whereas in MuSK-deficient mice neither AChR clusters nor examples of an arborized innervating motor neuron are observed (DeChiara et al., 1996). However, laminin- $\beta 2$ is unlikely to be the only MuSKinduced retrograde signal. The differentiation of the presynaptic nerve terminal is also affected in rapsyn-deficient mice (Gautam et al., 1995), despite the presence in the synaptic basal lamina of laminin- $\beta 2$ and agrin, which is also a stop signal for motor neurons (Campagna et al., 1995).

\section{MuSK-induced AChR clustering potentiated by muscle agrin}

Ectopic expression of a chick muscle cAgrin $_{7 \mathrm{A0B} 0}$ isoform facilitated, but was not essential for, AChR clusters induced by MuSKneuTMuSK. Because agrin does not directly interact with MuSK (Glass et al., 1996), the modulation of MuSK activity by muscle

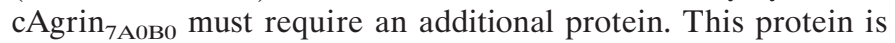
probably distinct from the putative agrin receptor, which is not recognized by isoforms of agrin lacking amino acid inserts at the B slice site (i.e., $B_{0}$ ) (Gesemann et al., 1996). A strong candidate for this role is $\alpha$-dystroglycan, which binds to both neural and muscle agrin (Bowe et al., 1994; Gee et al., 1994; Gesemann et al., 1996). In addition, agrin binding to $\alpha$-dystroglycan potentiates agrin-induced AChR clustering (Jacobson et al., 1998). Furthermore, $\beta$-dystroglycan, which associates with $\alpha$-dystroglycan and is central to the dystrophin-associated protein complex, was concentrated at MuSKneuTMuSK-induced AChR clusters (Fig. 5), as it is at the NMJ (Ervasti and Campbell, 1993).

\section{The requirement of MuSK for synaptic gene transcription}

Drawing together results obtained from innervated muscle fibers and cultured myotubes, it was concluded that AChR- $\epsilon$ subunit gene transcription, which at the NMJ occurs only from the subsynaptic nuclei in response to innervation (Brenner et al., 1990; Witzemann et al., 1991), was increased indirectly by MuSK via activation of the erbB/NRG pathway. This conclusion contrasts with data from neonatal rapsyn knock-out mice, in which MuSK remains accumulated and transcripts encoding AChR $\alpha$ - and $\gamma$-subunits are elevated, despite the absence of erbB receptors from the postsynaptic membrane (Gautam et al., 1995; Moscoso et al., 1995). However, high resolution in situ hybridization in neonatal rat muscle with AChR $\alpha$-subunit mRNA-specific probes shows that in developing endplate regions, the high level of $\alpha$-subunit mRNA, unlike $\epsilon$-subunit mRNA, may originate from unfused myoblasts (Brenner et al., 1990).

In summary, we have shown that activation of the MuSK kinase domain initiates a positive feedback loop whereby additional MuSK receptors are recruited, resulting in concentrated foci of MuSK that then induce AChR clusters. Although this process did not require neural agrin, inactive muscle agrin facilitated the activity of MuSKneuTMuSK. Because this agrin isoform does not bind the putative agrin receptor, different domains within agrin can independently modulate the activity of MuSK. Furthermore, MuSK is likely to be important in the differentiation of the innervating motor neuron (laminin- $\beta 2$ ) and later events in the maturation of the postsynaptic membrane (NaChs). Finally, MuSK indirectly activates synaptic gene transcription at the NMJ by assembly of the NRG/erbB pathway.

\section{REFERENCES}

Apel ED, Glass DJ, Moscoso LM, Yancopoulos GD, Sanes JR (1997) Rapsyn is required for MuSK signaling and recruits synaptic components to a MuSK-containing scaffold. Neuron 18:623-635.
Bargmann CI, Hung M-C, Weinberg R (1986) Multiple independent activations of the neu oncogene by a point mutation altering the transmembrane domain of p185. Cell 45:649-657.

Boudier J-L, Treut TL, Jover E (1992) Autoradiographic localization of voltage-dependent sodium channels on the mouse neuromuscular junction using $\mathrm{I}^{125}-\alpha$ scorpion toxin. II. Sodium channel distribution on postsynaptic membranes. J Neurosci 12:454-466.

Bowe MA, Deyst KA, Leszyk JD, Fallon JR (1994) Identification and purification of an agrin receptor from Torpedo postsynaptic membranes: a heteromeric complex related to the dystroglycans. Neuron 12:1173-1180.

Bowen DC, Park JS, Bodine S, Stark JL, Valenzuela DM, Stitt TN, Yancopoulos GD, Lindsay RM, Glass DJ, DiStefano PS (1998) Localization and regulation of MuSK at the neuromuscular junction. Dev Biol 199:309-319.

Brenner HR, Witzemann V, Sakmann B (1990) Imprinting of acetylcholine receptor message RNA accumulation in mammalian neuromuscular synapses. Nature 344:544-547.

Burke CL, Lemmon MA, Coren BA, Engelman DM, Stern DF (1997) Dimerization of the p185neu transmembrane domain is necessary but not sufficient for transformation. Oncogene 14:687-696.

Campagna JA, Ruegg MA, Bixby JL (1995) Agrin is a differentiationinducing "stop-signal" for motorneurons in vitro. Neuron 15: 1365-1374.

Colledge M, Froehner SC (1998) Signals mediating ion channel clustering at the neuromuscular junction. Curr Opin Neurobiol 8:357-363.

DeChiara TM, Bowen DC, Valenzuela DM, Simmons MV, Poueymirou WT, Thomas S, Kinetz E, Compton DL, Rojas E, Park JS, Smith C, DiStefano PS, Glass DJ, Burden SJ, Yancopoulos GD (1996) The receptor tyrosine kinase MuSK is required for neuromuscular junction formation in vivo. Cell 85:501-512.

Denzer AJ, Brandenberger R, Gesemann M, Chiquet M, Ruegg MA (1997) Agrin binds to the nerve-muscle basal lamina via laminin. J Cell Biol 137:671-683.

Ervasti JM, Campbell KP (1993) Dystrophin and the membrane skeleton. Curr Opin Cell Biol 5:82-86.

Ferns MJ, Campanelli JT, Hoch W, Scheller R, Hall Z (1993) The ability of agrin to cluster AChRs depends on alternative splicing and on cell surface proteoglycans. Neuron 11:491-502.

Fertuck HC, Salpeter MM (1976) Quantitation of junctional and extrajunctional acetylcholine receptors by electron microscope autoradiography after ${ }^{125} \mathrm{I}$-alpha bungarotoxin binding at the mouse neuromuscular junction. J Cell Biol 69:144-158.

Fischbach GD, Rosen KM (1997) ARIA: a neuromuscular junction neuregulin. Annu Rev Neurosci 20:429-458.

Gautam M, Noakes PG, Mudd J, Nichol M, Chu GC, Sanes JR, Merlie JP (1995) Failure of postsynaptic specialization to develop at neuromuscular junctions of rapsyn-deficient mice. Nature 377:232-236.

Gautam M, Noakes PG, Moscoso L, Rupp F, Scheller RH, Merlie JP, Sanes JR (1996) Defective neuromuscular synaptogenesis in agrindeficient mutant mice. Cell 85:525-535.

Gee SH, Montanaro F, Lindenbaum MH, Carbonetto S (1994) Dystroglycan- $\alpha$, a dystrophin-associated glycoprotein, is a functional agrin receptor. Cell 77:675-686.

Gesemann M, Denzer AJ, Ruegg MA (1995) Acetylcholine receptoraggregating activity of agrin isoforms and mapping of the active site. J Cell Biol 128:625-636.

Gesemann M, Cavalli V, Denzer AJ, Brancaccio A, Schumacher B, Ruegg MA (1996) Alternative splicing of agrin alters its binding to heparin, dystroglycan, and the putative agrin receptor. Neuron 16:755-767.

Glass DJ, Yancopoulos GD (1997) Sequential roles of agrin, MuSK and rapsyn during neuromuscular junction formation. Curr Biol 7:379-384.

Glass DJ, Bowen DC, Stitt TN, Radziejewski C, Bruno J, Ryan TE, Gies DR, Shah S, Mattsson K, Burden SJ, DiStefano PS, Valenzeula DM, DeChiara TM, Yancopoulos GD (1996) Agrin acts via a MuSK receptor complex. Cell 85:513-523.

Glass DJ, Apel ED, Shah S, Bowen DC, DeChiara TM, Stitt TN, Sanes JR, Yancopoulos GD (1997) Kinase domain of the muscle-specific receptor tyrosine kinase (MuSK) is sufficient for phosphorylation but not clustering of acetylcholine receptors: required role for the MuSK ectodomain? Proc Natl Acad Sci USA 94:8848-8853.

Hopf C, Hoch W (1998) Dimerization of the muscle-specific kinase induces tyrosine phosphorylation of acetylcholine receptors and their aggregation on the surface of myotubes. J Biol Chem 273:6467-6473. 
Jacobson C, Montanaro F, Lindenbaum M, Carbonetto S, Ferns M (1998) $\alpha$-Dystroglycan functions in acetylcholine receptor aggregation but is not a coreceptor for agrin-MuSK signaling. J Neurosci 18:6340-6348.

Jones G, Herczeg A, Ruegg MA, Lichtsteiner M, Kroger S, Brenner HR (1996) Substrate-bound agrin induces expression of acetylcholine receptor $\epsilon$-subunit gene in cultured mammalian muscle cells. Proc Natl Acad Sci USA, 93:5985-5990.

Jones G, Meier T, Lichtsteiner M, Witzemann V, Sakmann B, Brenner HR (1997) Induction by agrin of ectopic and functional postsynapticlike membrane in innervated muscle. Proc Natl Acad Sci USA 94:2654-2659.

Ko PK, Anderson MJ, Cohen MW (1977) Denervated skeletal muscle fibres develop discrete patches of high acetylcholine receptor density. Science 196:540-542.

Lupa MT, Krzemien DM, Schaller KL, Caldwell JH (1993) Aggregation of sodium channels during development and maturation of the neuromuscular junction. J Neurosci 13:1326-1336.

Martin PT, Sanes JR (1997) Integrins mediate adhesion to agrin and modulate agrin signalling. Development 124:3909-3917.

McMahan UJ (1990) The agrin hypothesis. Cold Spring Harb Symp Quant Biol 55:407-418.

Meier T, Hauser DM, Chiquet M, Landmann L, Ruegg MA, Brenner HR (1997) Neural agrin induces ectopic postsynaptic specializations in innervated muscle fibers. J Neurosci 17:6534-6544.

Meier T, Masciulli F, Moore C, Schoumacher F, Eppenberger U, Denzer AJ, Jones G, Brenner HR (1998a) Agrin can mediate AChR gene expression in muscle by aggregation of muscle-derived neuregulins. J Cell Biol 141:715-726.

Meier T, Marangi PA, Moll J, Hauser DM, Brenner H-R, Ruegg MA (1998b) A minigene of neural agrin encoding the laminin-binding and acetylcholine receptor-aggregating domains is sufficient to induce postsynaptic differentiation in muscle fibres. Eur $\mathrm{J}$ Neurosci 10:3141-3152.

Moscoso LM, Chu GC, Gautam M, Noakes PG, Merlie JP, Sanes JR (1995) Synapse-associated expression of an acetylcholine receptorinducing protein, ARIA/heregulin and its putative receptors, ErbB2 and ErbB3, in developing mammalian muscle. Dev Biol 172:158-169.

Patton BL, Miner JH, Chiu AY, Sanes JR (1997) Distribution and function of laminins in the neuromuscular system of developing, adult, and mutant mice. J Cell Biol 139:1507-1521.
Porter BE, Weis J, Sanes JR (1995) A motorneuron-selective stop signal in the synaptic protein S-laminin. Neuron 14:549-559.

Rimer M, Cohen I, Lømo T, Burden SJ, McMahan UJ (1998) Neuregulins and erbB receptors at neuromuscular junctions and at agrininduced postsynaptic-like apparatus in skeletal muscle. Mol Cell Neurosci 12:1-15.

Ruegg MA, Bixby JL (1998) Agrin orchestrates synaptic differentiation at the vertebrate neuromuscular junction. Trends Neurosci 21:22-27.

Ruegg MA, Tsim KWK, Horton SE, Kroger S, Escher G, Gensch EM, McMahan UJ (1992) The agrin gene codes for a family of basal lamina proteins that differ in function and distribution. Neuron 8:691-699.

Schmidt C, Lipsius E, Kruppa J (1995) Nuclear and nucleolar targeting of human ribosomal protein S6. Mol Biol Cell 6:1875-1885.

Siegel PM, Muller WJ (1996) Mutations affecting conserved cysteine residues within the extracellular domain of neu promote receptor dimerization and activation. Proc Natl Acad Sci USA 93:687-696.

Tinsley JM, Blake DJ, Zuellig RA, Davies KE (1994) Increasing complexity of the dystrophin-associated complex. Proc Natl Acad Sci USA 91:8703-8313.

Tzahar E, Pinkas-Kramarski R, Moyer JD, Klapper LN, Alroy I, Levkowitz G, Shelly M, Henis S, Eisenstein M, Ratzkin BJ, Sela M, Andrews GC, Yarden Y (1997) Bivalence of EGF-like ligands drives the ErbB signaling network. EMBO J 16:4938-4950.

Umesono K, Murakami KK, Thompson CC, Evans RM (1991) Direct repeats as selective response elements for the thyroid hormone, retinoic acid, and vitamin D3 receptors. Cell 65:1255-1266.

Valenzuela DM, Stitt TN, DiStefano PS, Rojas E, Mattsson K, Compton DL, Nunez L, Park JS, Stark JL, Gies DR, Thomas S, Beau MML, Fernald AA, Copeland NG, Jenkins NA, Burden SJ, Glass DJ, Yancopoulos GD (1995) Receptor tyrosine kinase specific for the skeletal muscle lineage: expression in embryonic muscle, and at the neuromuscular junction, and after injury. Neuron 15:573-584.

Wallasch C, Weiss FU, Niederfellner G, Jallal B, Issing W, Ullrich A (1995) Heregulin-dependent regulation of HER2/neu oncogenic signalling by heterodimerization ith HER3. EMBO J 14:4267-4275.

Witzemann V, Brenner HR, Sakmann B (1991) Neural factors regulate AChR subunit mRNAs at rat neuromuscular junctions $\mathrm{J}$ Cell Biol 114:125-141.

Xie M-H, Yuan J, Adams C, Gurney A (1997) Direct demonstration of MuSK involvement in acetylcholine receptor clustering through identification of agonist ScFv. Nat Biotechnol 15:768-771. 